\title{
CYCLE-ANNUEL D'ACTIVITÉ DE LA TRUITE COMMUNE (SALMO TRUTTA L.) ADULTE : ÉTUDE PAR RADIO-PISTAGE DANS UN COURS D'EAU DE L'ARDENNE BELGE.
}

\author{
M. OVIDIO \\ Université de Liège, Institut de Zoologie, Laboratoire de Démographie des Poissons \\ et d'Aquaculture, 10 Chemin de la Justice, 4500 Tihange, Belgique. \\ E-mail : M.Ovidio@ulg.ac.be
}

\section{RÉSUMÉ}

Sur une durée totale d'expérience de vingt-six mois, vingt truites $(26,0-57,0 \mathrm{~cm} \mathrm{LF}$; 198-1 $685 \mathrm{~g}$ ) ont été quotidiennement suivies par radio-pistage dans l'Aisne (affluent de l'Ourthe) durant des périodes variant de 466 à 16 jours selon les individus et les contraintes techniques expérimentales, notamment la durée de vie des batteries. II apparaît que l'amplitude et la fréquence des mouvements sont le plus élevées d'octobre à décembre, pendant la période de reproduction. Les migrations vers l'amont (max. : $25 \mathrm{~km}$ ) ont principalement lieu en octobre et pendant la première quinzaine de novembre et sont déclenchées par des variations combinées du niveau d'eau et de la température, dans une gamme thermique précise $\left(10^{\circ} \mathrm{C}\right.$ a $\left.14^{\circ} \mathrm{C}\right)$. Le frai (de la deuxième quinzaine de novembre à la fin décembre) a lieu dans le cours principal (largeur maximale : $10 \mathrm{~m}$ ) ou dans de petits affluents. Les truites qui survivent effectuent un homing post-reproducteur rapide (max. : $9200 \mathrm{~m}$ en $24 \mathrm{~h}$ ) et ṕrécis. En dehors du contexte de la reproduction, les déplacements sont-moins longs et correspondent essentiellement à des changements alternés de gîtes dans un domaine vital bien défini dont la longueur est corrélée avec la taille des truites. En mars et juin, certaines truites effectuent des migrations unidirectionnelles de grande amplitude vers l'amont dont la signification biologique est inconnue. Les résultats sont discutés dans le contexte des stratégies de vie de la truite et de la gestion des populations.

Mots-clés : mouvement, migration, gestion de population, stratégie de vie, barrage, truite, Salmo trutta, bassin de la Meuse, Belgique.

\section{ANNUAL ACTIVITY CYCLE OF ADULT BROWN TROUT (SALMO TRUTTA L.) : A RADIO-TELEMETRY STUDY IN A SMALL STREAM OF THE BELGIAN ARDENNE.}

\begin{abstract}
During a study period of 26 months, twenty trout $(26.0-57.0 \mathrm{~cm}$ FL ; 198-1,685 g) were daily located from 16 to 466 days in a small stream of the Belgian Ardenne, the Aisne stream (tributary of the river Ourthe) in order to characterize their annual pattern of mobility.
\end{abstract}


Daily movements were more frequent and longer during the spawning period (October-December) than at any other time of the year. Upstream migrations (max. : $25 \mathrm{~km}$ ) generally occurred during October and the first fortnight of November and were triggered by a combination of variations of water temperature and water level within a thermal range of $10-14^{\circ} \mathrm{C}$. Spawning activity (second fortnight of November until late December) took place in the Aisne stream (max. width : $10 \mathrm{~m}$ ) or in its small tributaries. Trout surviving spawning undertook a fast (max. : 9,200 $\mathrm{m}$ in $24 \mathrm{~h}$ ) and precise post reproductive homing. From winter to summer, daily movements were shorter and mainly corresponded to changes of residences within a home range of which the size was proportional to the trout's size. In March and June, some trout made long upstream unidirectional migrations of which the biological signification is still unknown. These results are discussed within the context of life history strategies and management of trout populations.

Key-words : movement, migration, management of population, life history strategy, weir, trout, Salmo trutta, River Meuse basin, Belgium.

\section{INTRODUCTION}

Les phénomènes de mobilité et de dispersion (mobilité journalière, domaine vital, migration de reproduction, comportement de homing) et leurs variations au cours du cycle annuel sont des éléments majeurs de la biologie des populations de poissons, aux plans de leur démographie et de leur productivité (NORTHCOTE, 1967 ; PHILIPPART, 1975), de leur génétique (ALLENDORF, 1996) et de leur gestion halieutique (MAISSE et BAGLINIĖRE, 1991 ; LARINIER et al., 1995). Compte tenu des contraintes environnementales sur les méthodologies d'échantillonnage (e.g. pêche à l'électricité ou observation directe), la biotélémétrie constitue une méthode d'investigation adéquate pour la caractérisation de l'ensemble de ces paramètres de mobilité et de dispersion, principalement chez les individus adultes. Les études couvrant l'ensemble d'un cycle annuel sont rares en biotélémétrie des poissons (BARAS, 1992 chez Barbus barbus; MEYERS et al., 1992 chez S. trutta ; JAMES et KELSO, 1985 chez Oncorhynchus mykiss).

En Amérique du Nord, où elle a été introduite, la truite commune a déjà fait l'objet de quelques études par radio-pistage (CLAPP et al., 1990 ; MEYERS et al., 1992 ; YOUNG, 1994). En revanche, les patrons de mobilité de $S$. trutta dans les populations européennes n'ont jamais été étudiés sur l'ensemble d'un cycle annuel. Cette caractérisation est d'autant plus indispensable et urgente que tendent à se développer les rempoissonnements avec des souches d'élevage, susceptibles de modifier à terme les stratégies d'occupation du temps et de l'espace des souches locales (GUYOMARD et KRIEG, 1986) et que le caractère résident, en dehors de la période de reproduction, des Salmonidés de rivière, et notamment des truites communes adultes, est remis en cause par plusieurs études (GOWAN et al., 1994 ; FAUSH et YOUNG, 1995 ; OVIDIO et al., in press). Par ailleurs, il existe encore de nombreux types d'obstacles et barrages dont l'impact sur la libre circulation des poissons reste inconnu (OVIDIO et al., 1996).

L'objectif de cet article est de présenter une synthèse sur la mobilité de 20 truites adultes radio-pistées de 466 à 16 jours sur une durée d'expérience de 26 mois, et de déterminer les variations saisonnières des patrons de mobilité et d'utilisation du domaine vital. Les problèmes d'utilisation du microhabitat (nycthéméral et entre jours successifs) et des cycles d'activités journaliers seront envisagés dans de futures publications (OVIDIO, recherches doctorales en cours, étude des stratégies individuelles d'utilisation spatio-temporelle des ressources chez la truite commune $S$. trutta). 
De manière à aborder le problème dans une situation aussi naturelle que possible, nous avons choisi comme système de référence l'Aisne, petite rivière ardennaise salmonicole, bien préservée des interventions anthropiques, et où se déroulent depuis 1979 des recherches à long terme sur la dynamique des populations de l'ombre et de la truite (PHILIPPART, 1998). L'étude porte sur des truites de rivière, les populations anadromes étant en cours de restauration démographique dans le bassin de la Meuse (PHILIPPART, 1987.; PHILIPPART et al., 1994 ;.P.HILIPPART. et al., 1997).

\section{SITE D'ÉTUDE}
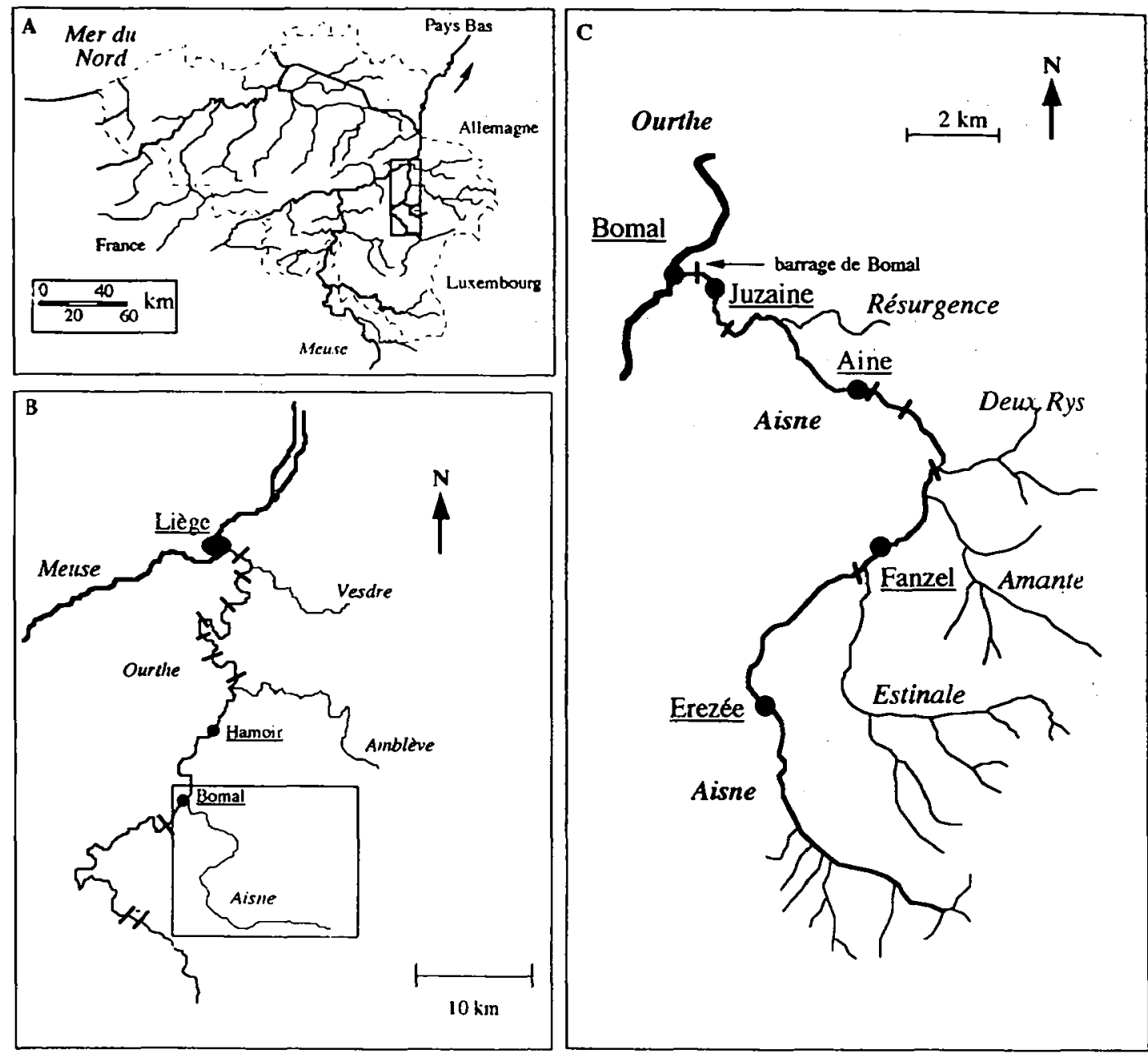

Figure 1

Localisation du site d'étude, le sous-bassin de l'Aisne (affluent de l'Ourthe) entre Bomal et Erezée. Les barres transversales sur le tracé de la rivière représentent les petits déversoirs et barrages qui pourraient perturber les déplacements des truites.

\section{Figure 1}

Localisation of the study area, the Aisne Stream sub-basin (tributary of the River Ourthe) in between Bomal and Erezée in the Belgian Ardennes. Transverse bars are small weirs which may interfere with the free circulation of fish. 
L'étude s'est déroulée dans l'Aisne (bassin de la Meuse, sous-bassin de l'Ourthe), affluent de l'Ourthe à Bomal (Figures $1 \mathrm{~A}$ et 1B). L'Aisne est une rivière salmonicole à lit caillouteux qui prend sa source à $600 \mathrm{~m}$ d'altitude. La pente et le débit moyens sont respectivement de $2,78 \%$ et de $2,43 \mathrm{~m}^{3} . \mathrm{s}^{-1}$ et la largeur dépasse rarement une dizaine de mètres. La température de l'eau varie entre 0 et $19^{\circ} \mathrm{C}$ avec une moyenne annuelle de $9,4^{\circ} \mathrm{C}$. Le peuplement de poissons est typique de la zone à ombre (Huet, 1949). II est principalement constitué de la truite de rivière (Saimo trutta), de. l'ombre commun (Thymallus thymallus), du saumon atlantique juvénile (Salmo salar) réintroduit dans le cadre du projet "Meuse Saumon 2000" (PHILIPPART et al., 1994), du chabot (Cottus gobio), de la loche franche (Barbatula barbatula) et de l'anguille (Anguilla anguilla). Les principaux affluents de l'Aisne sont le Deux-Rys, l'Amante et l'Estinale (Figure 1C).

Sur les six petits déversoirs présents sur son cours, seul celui de Bomal, situé à $500 \mathrm{~m}$ en amont du confluent avec l'Ourthe, est équipé depuis fin 1995 d'une passe à poissons à bassins successifs (Figure 1C).

\section{MATÉRIEL ET MÉTHODES}

Vingt truites (Tableau I) ont été capturées dans l'Aisne par pêche à l'électricité (générateur DEKA 5000) ou lors des contrôles du piège de l'échelle à poissons du barrage de Bomal près du confluent de l'Aisne avec l'Ourthe (Figure 1C). Elles ont été équipées d'émetteurs radio (< $2 \%$ du poids corporel, $40 \mathrm{MHz}$, A.T.S., Inc) implantés dans la cavité intrapéritonéale via une incision médioventrale pratiquée en arrière de la ceinture pelvienne, sous anesthésie, suivant la procédure décrite par BARAS et al. (in press).

Les poissons étaient relâchés à l'endroit précis de capture dès qu'ils retrouvaient leur équilibre spontané et leur capacité de nage (environ $3 \mathrm{~min}$ après l'opération chirurgicale). Ils ont été localisés quotidiennement (entre $9 \mathrm{~h}$ et $12 \mathrm{~h}$ ) du mois d'octobre 1995 au mois de février 1998 (1655 localisations, récepteur radio Fieldmaster, antenne diamant, A.T.S., Inc). En complément, plusieurs suivis nocturnes ont été réalisés au cours de l'étude. Le nombre de poissons étudiés à chaque saison est de huit au printemps, onze en été, quatorze en automne et neứ en hiver, certains individus ayant été pistés pendant plusieurs saisons consécutives. La précision des localisations (biangulation à partir de balises installées sur les berges) était de 1 à $2 \mathrm{~m}^{2}$ selon la distance et la largeur de la rivière.

La mobilité des truites a été caractérisée par des indicateurs à différentes échelles spatiales, définis ci-dessous :

- mouvement journalier net (MJN) : indicateur de mobilité spatiale correspondant à la distance séparant deux localisations espacées de $24 \mathrm{~h}$;

- domaine vital (DV) : aire occupée par un individu y développant l'ensemble de ses activités. II est exprimé par son extension longitudinale déterminée par la distance entre la localisation la plus en amont et celle la plus en aval (BARAS, 1992). Pour un même individu, il peut être calculé à différentes échelles temporelles (journalier, mensuel, saisonnier, annuel) et peut donc contenir (selon l'échelle temporelle choisie) l'aire de reproduction ;

- gîte : zone de surface réduite dans laquelle la truite est localisée avec la plus grande fréquence. Elle peut y développer une activité ou être au repos. Le gîte estival est considéré comme le gîte occupé en dehors de la période de reproduction ;

- séquence de faciès : succession des faciès d'écoulement, souvent marquée par l'alternance radier/profond (MALAVOI, 1989 in HAURY et al., 1991). 
La température était enregistrée en continu (enregistreurs TidBIT, ONSET Inc.) et la hauteur d'eau mesurée quotidiennement sur une échelle limnimétrique installée à Bomal-Juzaine (précisions respectives de $0,1^{\circ} \mathrm{C}$ et $1 \mathrm{~cm}$ ). La turbidité de l'eau était estimée à vue par un système de codes : $1=$ limpide ; 2 = légèrement trouble ; $3=$ trouble ; $4=$ très trouble.

Les relations entre les variables environnementales et la mobilité des truites ont été analysées par régressions multiples pas à pas, analyses de variance (1 way- et 2 way-ANOVA) et tables de contingences.

\section{Tableau I}

Caractéristiques des truites radio-pistées et périodes de suivi.

\section{Table I}

Characteristics and tracking periods of the twenty radio-tagged brown trout.

\begin{tabular}{cccccc}
\hline Truites & $\begin{array}{c}\text { Taille/LF. } \\
(\mathbf{c m})\end{array}$ & $\begin{array}{c}\text { Poids } \\
(\mathbf{g})\end{array}$ & Sexe & Période de suivi & $\begin{array}{c}\text { Durée } \\
\text { du suivi (j) }\end{array}$ \\
\hline 1 & 39,9 & 611 & $M$ & $12 / 10 / 95-10 / 08 / 96$ & 302 \\
2 & 28,5 & 275 & M & $22 / 04 / 96-31 / 07 / 97$ & 466 \\
3 & 27,9 & 234 & & $03 / 06 / 96-12 / 08 / 96$ & 70 \\
4 & 42,0 & 995 & $M$ & $20 / 06 / 96-16 / 07 / 96$ & 26 \\
5 & 28,0 & 305 & & $14 / 08 / 96-01 / 12 / 96$ & 107 \\
6 & 28,8 & 271 & & $14 / 08 / 96-11 / 05 / 97$ & 271 \\
7 & 26,6 & 233 & & $14 / 08 / 96-02 / 11 / 96$ & 80 \\
8 & 27,2 & 233 & $M$ & $14 / 08 / 96-24 / 12 / 96$ & 132 \\
9 & 29,5 & 287 & & $19 / 09 / 96-12 / 12 / 96$ & 84 \\
10 & 42,8 & 755 & & $25 / 02 / 97-28 / 09 / 97$ & 215 \\
11 & 34,7 & 380 & $F$ & $05 / 03 / 97-26 / 03 / 97$ & 21 \\
12 & 30,4 & 288 & & $20 / 03 / 97-09 / 10 / 97$ & 203 \\
13 & 29,9 & 290 & $F$ & $18 / 04 / 97-30 / 12 / 97$ & 256 \\
14 & 26,3 & 206 & $F$ & $22 / 09 / 97-24 / 10 / 97$ & 32 \\
15 & 28,7 & 285 & $M$ & $22 / 09 / 97-19 / 10 / 97$ & 27 \\
16 & 31,2 & 348 & $F$ & $22 / 09 / 97-08 / 10 / 97$ & 16 \\
17 & 26,5 & 198 & M & $22 / 09 / 97-18 / 10 / 97$ & 26 \\
18 & 26,0 & 206 & $F$ & $22 / 09 / 97-20 / 10 / 97$ & 28 \\
19 & 57,0 & 1685 & & $30 / 10 / 97-05 / 01 / 98$ & 67 \\
20 & 40,2 & 681 & M & $23 / 10 / 97-23 / 02 / 98$ & 123 \\
\hline
\end{tabular}

\section{RÉSULTATS}

\section{Migration de reproduction automnale}

Les migrations de reproduction ont principalement lieu du mois d'octobre au mois de décembre. Proportionnellement à la longueur de l'Aisne $(40 \mathrm{~km})$, les distances parcourues sont grandes (moyenne: $8450 \mathrm{~m}$; maximum : $24790 \mathrm{~m}$, Figure 2 et Tableau II). Les migrations vers les frayères durent de 1 à 15 jours. Lors de la montaison, les mouvements journaliers nets (MJN) peuvent atteindre $7200 \mathrm{~m}$. Les migrations sont presque exclusivement nocturnes, mais, à trois reprises, nous les avons également observées en plein jour. 


\section{Tableau II}

Caractéristiques des migrations effectuées par les truites suivies pendant la période de reproduction.

\section{Table II}

\section{Characteristics of the spawning migrations of brown trout in the Aisne Stream.}

\begin{tabular}{cccl}
\hline $\begin{array}{c}\text { truite } \\
n^{*}\end{array}$ & $\begin{array}{c}\text { Iongueur de la } \\
\text { migration } \\
(\mathbf{k m})\end{array}$ & $\begin{array}{c}\text { durée de la } \\
\text { migration } \\
(\mathbf{j})\end{array}$ & remarques \\
\hline 1 & 0,89 & 1 & homing en 1 jour \\
5 & 4,25 & 3 & expulsion de l'émetteur pendant le frai \\
6 & 2,20 & 2 & perdue puis retouvée en été dans un gíte estival (homing) \\
7 & 21,28 & $15^{(-)}$ & perdue pendant la migration \\
8 & 24,79 & 12 & pile de l'émetteur usée sur le site de frai \\
9 & 14,26 & 14 & pile de l'émetteur usée sur le site de frai \\
11 & 6,65 & $8^{(-)}$ & perdue pendant la migration \\
12 & 5,02 & $2^{(*)}$ & perdue pendant la migration \\
13 & 9,58 & 12 & homing en 1 jour \\
14 & 2,52 & $2^{(\cdot)}$ & pile de l'émetteur usée pendant la migration \\
15 & 11,01 & $10^{(*)}$ & pile de l'émetteur usée pendant la migration \\
16 & 2,43 & $1^{(*)}$ & pile de l'émetteur usée pendant la migration \\
19 & 5,02 & 2 & homing en 2 jours \\
\hline
\end{tabular}

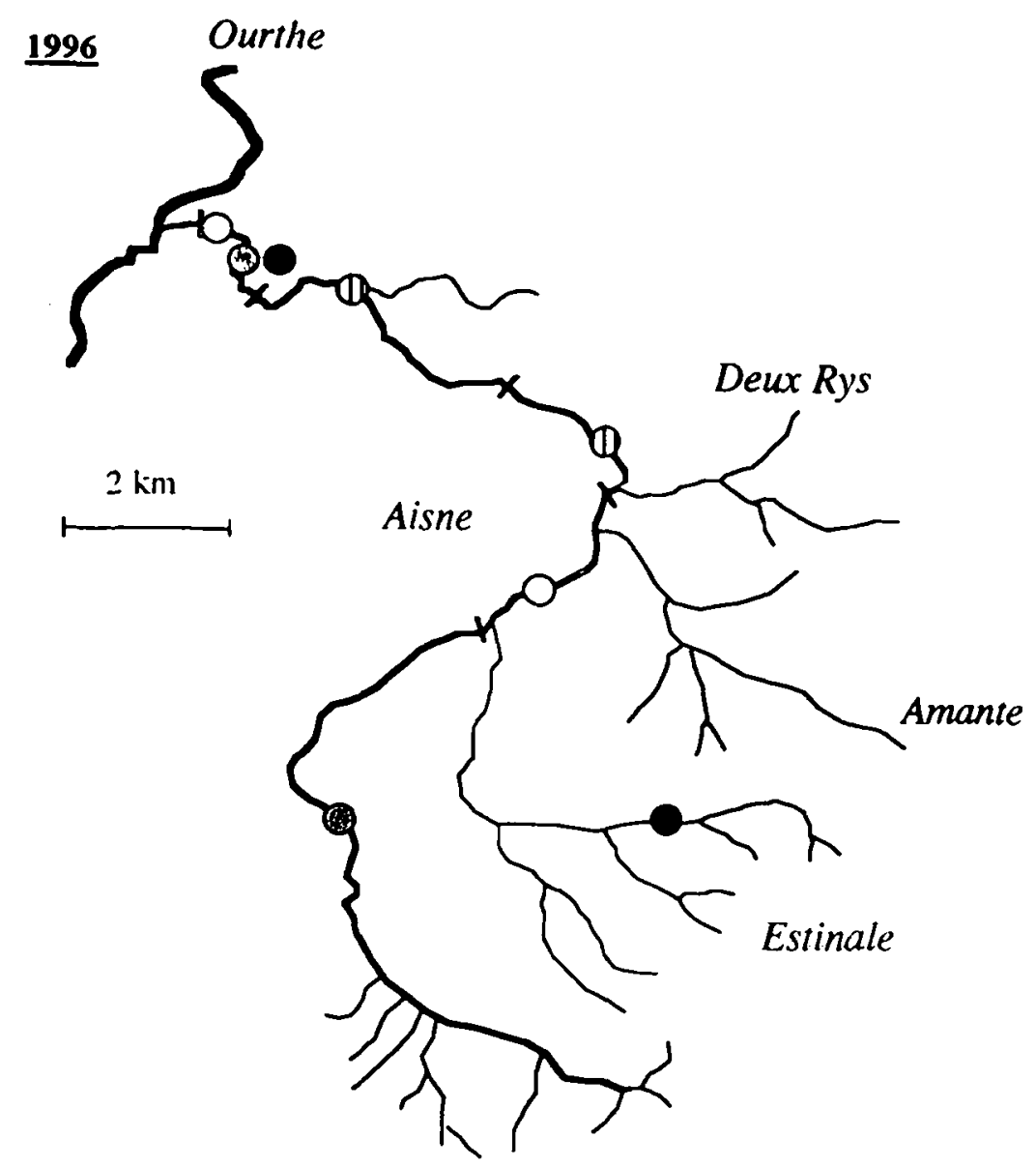




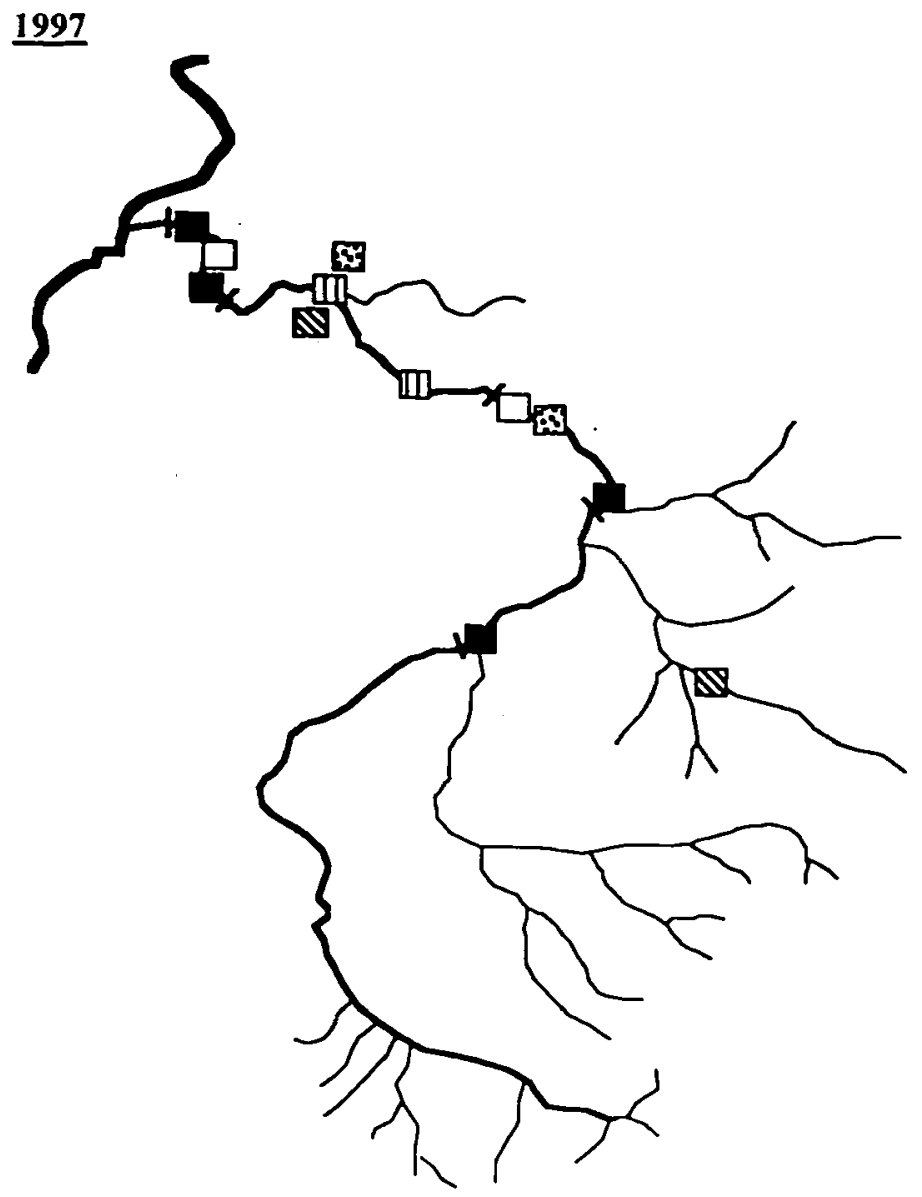

Figure 2

Migrations de reproduction des truites dans l'Aisne et ses affluents durant l'automne 1996 et 1997. Deux symboles identiques représentent les limites aval et amont de la migration pour un même individu.

\section{Figure 2}

Spawning migrations of brown trout in the Aisne Stream and its tributaries during the autumn in 1996 and 1997. Two identical symbols represent the downstream and upstream limits of the migration for one trout.

Souvent, les patrons de progression des truites sont comparables à des courbes logarithmiques : les MJN observés les premiers jours sont de plus grandes amplitudes et plus fréquents. Au fur et à mesure que la truite s'approche des frayères, la fréquence et l'amplitude des déplacements diminuent (Figure 3). Le frai peut avoir lieu dans le cours principal de l'Aisne, mais de très petits affluents sont aussi empruntés et remontés parfois sur de longues distances (Figure 2). Le temps passé sur les sites de frai varie selon les individus de un jour à plus d'un mois. Lorsqu'elle a pu être observée, la dévalaison était toujours plus rapide que la montaison (max. : $9150 \mathrm{~m}$ en $24 \mathrm{~h}$, Figure 3) et avait toujours lieu du mois de novembre au mois de janvier. 


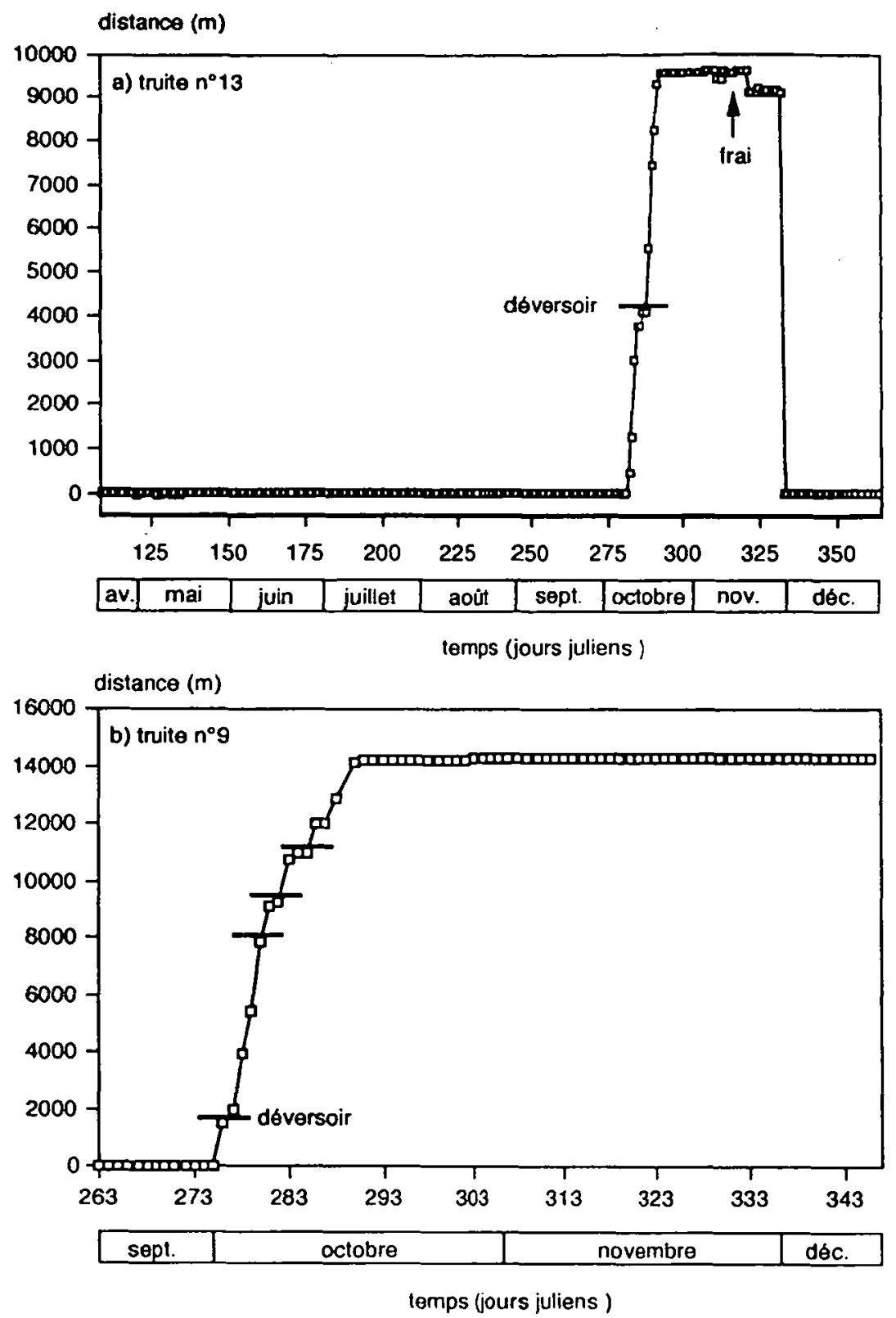

Figure 3

Exemples de migration de reproduction (a) truite $n^{\circ} 13$ : la migration d'une durée de 12 jours commence le 9 octobre, le frai a lieu à la mi-novembre et le homing (en 1 jour) en fin novembre. Lors de la montaison, un déversoir (trait horizontal) retarde la truite pendant 4 jours. (b) truite $n^{\circ} 9$; la migration d'une durée de 14 jours commence le 2 octobre, deux des quatre déversoirs retardent la migration ; le frai ne peut être observé à cause de la forte turbidité de l'eau.

\section{Figure 3}

Examples of spawning migrations (a) trout $n^{\circ} 13:$ the 12 days migration started on 9 October, spawning took place in mid-November and the fish homed in one day in late November. During the upstream migration, a small weir (horizontal line) delayed the migration by 4 days (b) trout $n^{\circ} 9$ : left its summer home range on 2 October and moved upstream during 14 days. Two of the four weirs on its way caused short delays during the upstream migration. High turbidity in the stream prevented any direct observation of trout spawning. 
Domaine vital et gîte hors période de reproduction

Tableau III

Valeurs des paramètres de mobilité et de dispersion des truites suivies pendant le printemps et l'été.

\section{Table III}

Value of the mobility and dispersion parameters of the radio-tagged brown trout during spring and summer in the Aisne Stream.

\begin{tabular}{ccccc}
\hline $\begin{array}{c}\text { Truite } \\
\mathbf{n}^{\circ}\end{array}$ & $\begin{array}{c}\text { Longueur } \\
\text { fourche } \\
(\mathbf{c m})\end{array}$ & $\begin{array}{c}\text { Domaine } \\
\text { vital } \\
(\mathbf{m})\end{array}$ & $\begin{array}{c}\text { Nombre } \\
\text { de gîtes }\end{array}$ & $\begin{array}{c}\text { Dist. totale } \\
\text { parcourue } \\
(\mathbf{m})\end{array}$ \\
\hline 1 & 39,9 & 144 & 5 & 458 \\
2 & 30,5 & 75 & 2 & 458 \\
3 & 27,9 & 15 & 2 & 97 \\
4 & 42,0 & 4700 & 7 & 5300 \\
5 & 28,0 & 141 & 3 & 1512 \\
6 & 28,8 & 85 & 1 & 288 \\
7 & 26,6 & 214 & 2 & 883 \\
8 & 27,2 & 90 & 1 & 462 \\
10 & 42,8 & 354 & 5 & 3139 \\
12 & 30,4 & 327 & 4 & 430 \\
13 & 29,9 & 43 & 2 & \\
\hline
\end{tabular}

Au printemps et en été, la dimension du domaine vital (DV) des onze truites radio-pistées dans l'Aisne varie entre $15 \mathrm{~m}$ et $4700 \mathrm{~m}$ (Tableau III). Comme peu d'individus appartenant à des classes de taille différentes ont été suivis, une certaine prudence s'impose dans l'interprétation des résultats ci-dessous. II apparaît que l'élendue du DV est corrélée avec la longueur des individus $\left(p=0,0336 ; R^{2}=0,411 ; 10 \mathrm{DL}\right.$; Figure 4) mais qu'elle est indépendante du nombre de jours de suivi ( $p>0,05,10 \mathrm{DL}$ ). La nature semi-logarithmique de la relation entre le domaine vital et la mobilité totale ( $p=0,0001, R^{2}=0,83,10 \mathrm{DL} ;$ Figure 4 ) indique que les truites n'exploitent pas leur domaine vital printanier et estival de manière séquentielle : elles sont fidèles à un gîte ou en utilisent plusieurs en alternance comme le montrent les exemples de la Figure 5.

Les truites sont très attachées à un ou plusieurs gîtes, espacés de quelques mètres à plusieurs centaines de mètres (Figure 5). Le nombre moyen de gîtes est de 3 (min.: 1 ; max. : 7 ; Tableau III) et est positivement et significativement corrélé avec la taille des individus ( $p=0,0004 ; R^{2}=0,775 ; 10 \mathrm{DL}$, Figure 4) et avec celle de leur domaine vital $\left(p=0,005 ; R^{2}=0,630 ; 10 \mathrm{DL} ;\right.$ Figure 4). Des suivis réalisés en continu, sur une période de $24 \mathrm{~h}$ (OVIDIO et al., résultats non publiés), ont montré que les gîtes occupés pendant les localisations diurnes peuvent correspondre à des milieux où la truite s'alimente ou se repose.

\section{Probabilité annuelle de mouvement}

Au cours de l'année, la probabilité de réaliser des mouvements supérieurs à $40 \mathrm{~m}$ (longueur moyenne approximative d'une séquence de faciès dans l'Aisne) est assez variable (Figure 6). Les analyses par tables de contingences indiquent que leur répartition diffère significativement d'une distribution homogène $\left(p<0,0001 ; X^{2}=77,13 ; D L=23\right)$. 
La probabilité moyenne annuelle de changement de séquence est de $12 \%$. L'évolution de la courbe des probabilités des mouvements supérieurs à $5 \mathrm{~m}$ (Figure 6) est assez semblable à celle des mouvements supérieurs à $40 \mathrm{~m}$ et est aussi significativement hétérogène $\left(p<0,0001 ; X^{2}=60,62 ; D L=23\right)$. Les fréquences des mouvements sont largement supérieures et la probabilité annuelle d'effectuer ce genre de déplacements (> $5 \mathrm{~m}$ ) est de 29,6\% (presque un jour sur trois). Les truites sont donc relativement mobiles toute l'année, mais les mouvements de grande amplitude sont peu fréquents en dehors de la période de reproduction.
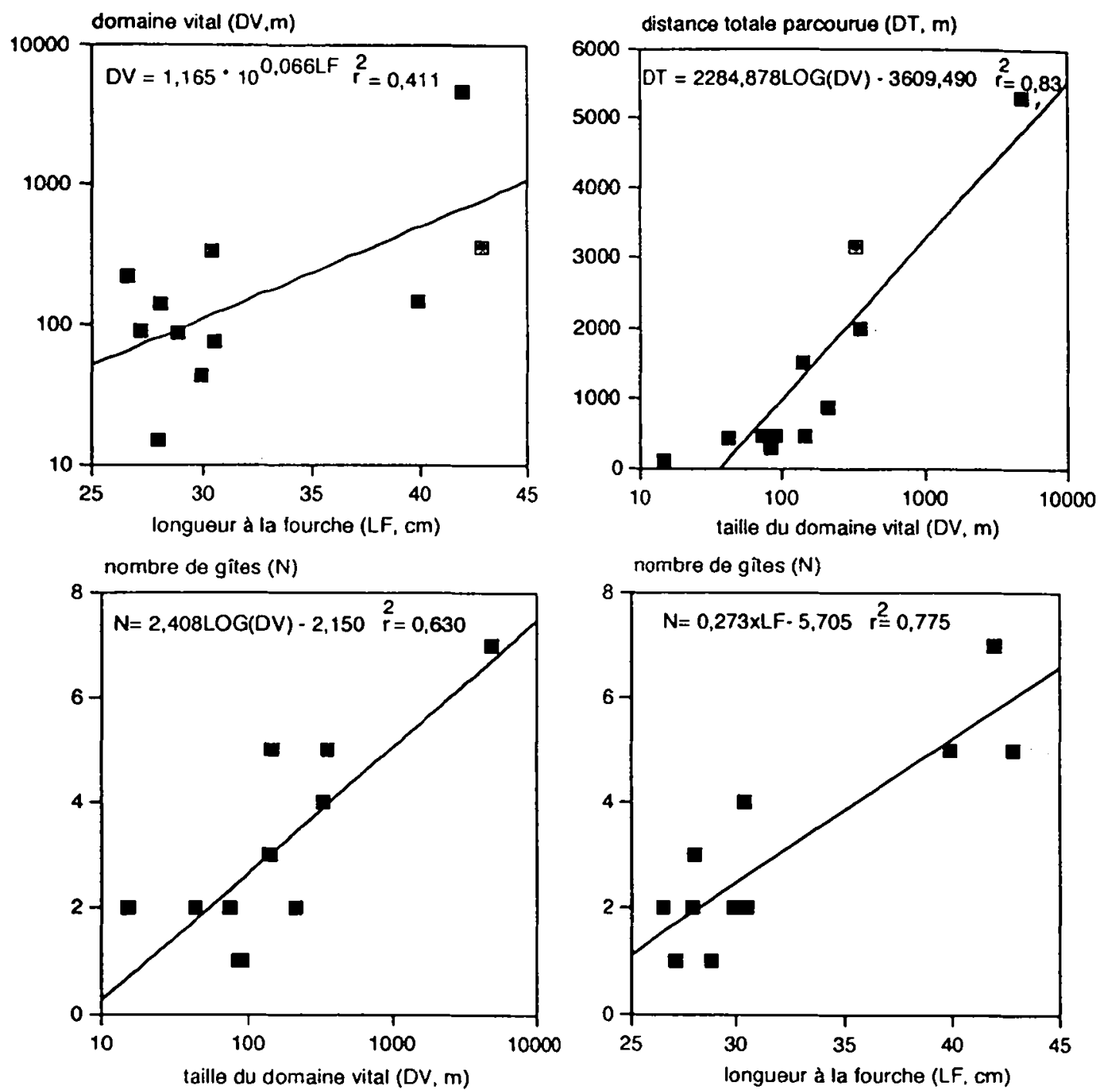

Figure 4

Relations entre différents paramètres de mobilité et de dispersion chez les truites de l'Aisne (21 mars-21 septembre).

Figure 4

Relationships between some mobility and dispersion parameters of brown trout during spring and summer in the Aisne Stream. 
localisation (m)

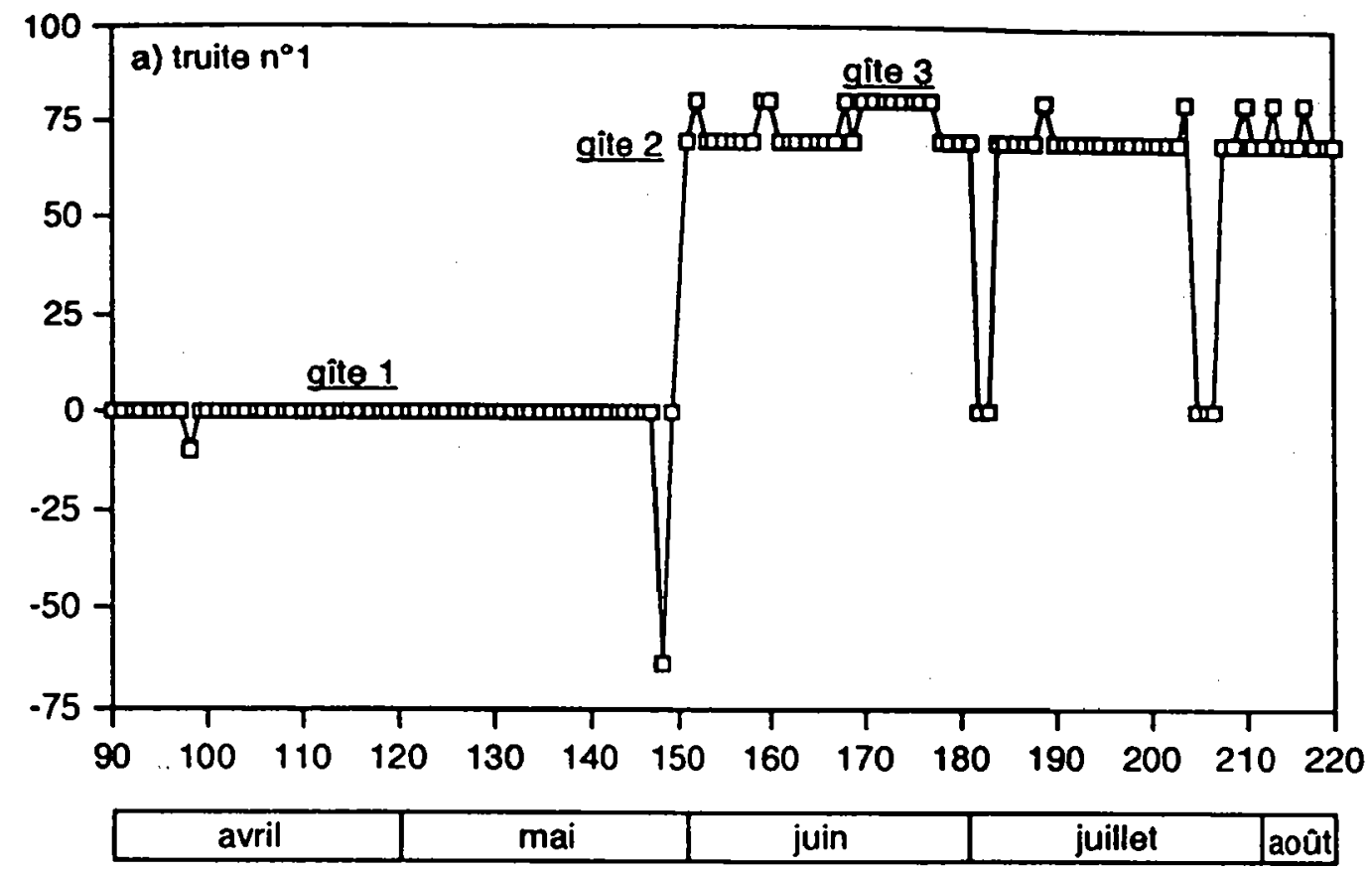

temps (jours juliens)

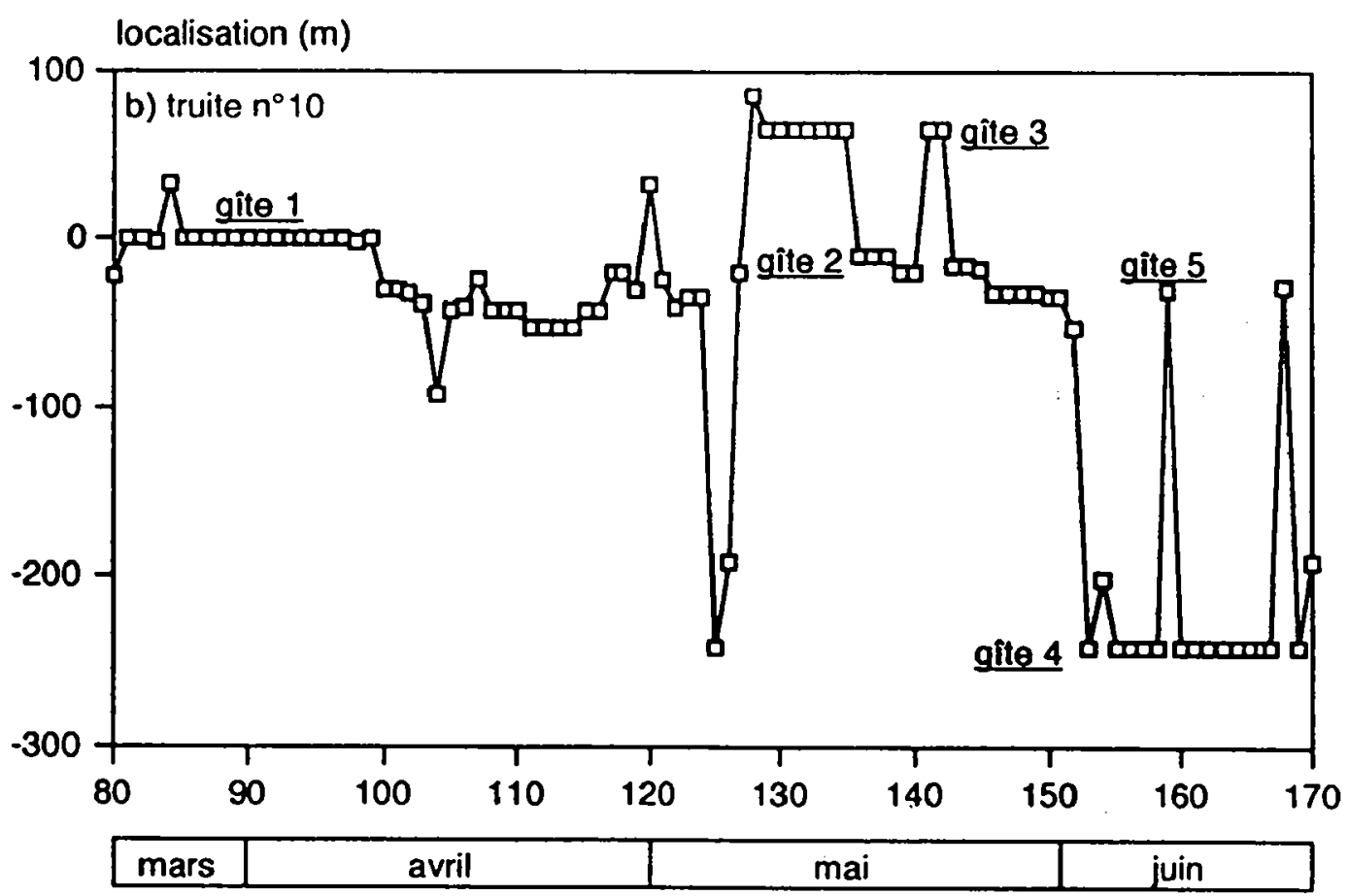

temps (jours juliens)

Figure 5

Exemples d'occupation alternée de gîtes durant la période printanière et estivale chez les truites $n^{\circ} 1$ et $n^{\circ} 10$. Poissons capturés et remis à l'eau au point 0 .

Figure 5

Examples of alternate use of resting places during spring and summer by brown trout $n^{\circ} 1$ (a) and $n^{\circ} 10$ (b). 


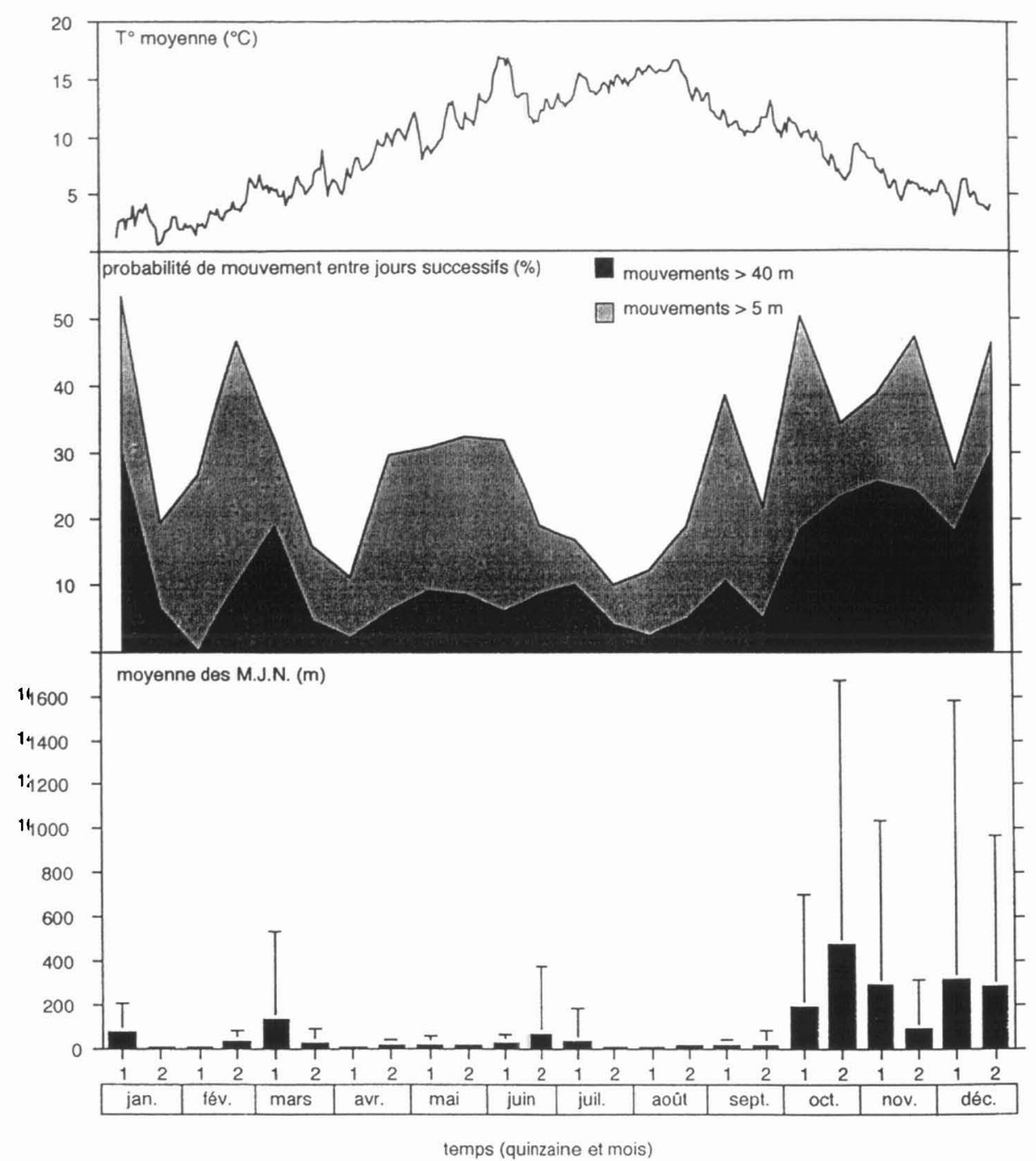

Figure 6

Variation, par quinzaines de jours, des mouvements nets journaliers (MJN : distance entre deux localisations espacées de $24 \mathrm{~h}$ ) et de la probabilité (nombre de mouvements/nombre de mouvements possibles) de réaliser des mouvements supérieurs à $\mathbf{5} \mathrm{m}$ et à $\mathbf{4 0} \mathrm{m}$ entre jours successifs.

\section{Figure 6}

Variation, by fortnight, of the net daily journeys (NDJ : distance between two consecutive daily locations) and the probability (number of movements/number of possible movements) of moving further than $5 \mathrm{~m}$ and $40 \mathrm{~m}$ between consecutive days.

\section{Analyse causale des patrons saisonniers de mobilité}

Pour le déclenchement des migrations de reproduction, l'influence des facteurs environnementaux est déterminante. Deux années de suivi ont permis de préciser que les migrations débutent presque exclusivement lors de variations combinées de la température (chute ou hausse) et du niveau d'eau (baisse ou augmentation), dans une plage thermique de 10 à $14^{\circ} \mathrm{C}$ et en photopériode décroissante. Ces conditions 
environnementales étant rencontrées à plusieurs reprises en automne, tous les individus ne débutent pas leur migration exactement le même jour et il en résulte une migration par vagues successives (OVIDIO et al., 1998 pour plus de détails).

En dehors du contexte des migrations de reproduction, il existe une relative variabilité de l'influence des facteurs température, niveau d'eau et turbidité suivant l'individu. considéré. Cette variabilité peut suggérer qu'en dehors de la reproduction, ce sont d'autres facteurs qui prévalent.

On notera aussi que les fortes crues (débit $>15 \mathrm{~m}^{3} \cdot \mathrm{s}^{-1}$ ) génèrent, chez presque toutes les truites étudiées, une mobilité accrue et une occupation inhabituelle de l'espace qui prend rapidement fin lorsque les niveaux d'eau redeviennent normaux.

\section{DISCUSSION ET CONCLUSION}

Pour les suivis à long terme, le radio-marquage des truites adultes par implantation chirurgicale de l'émetteur dans la cavité abdominale est actuellement reconnu comme étant la méthodologie la plus fiable (BARAS et al., in press) et est utilisée dans les études les plus récentes (CLAPP et al., 1990 ; MEYERS et al., 1992 ; YOUNG, 1994 ; OVIDIO et al., in press; OVIDIO et al., 1998). Dans notre cas, une étude préliminaire réalisée sur une souche de truite ardennaise en rivière artificielle (BIRTLES et al., 1995) a permis de confirmer l'adéquation de la procédure d'implantation chirurgicale, notamment au plan de la rétention à long terme de l'émetteur et de la perturbation comportementale. De plus, à cinq reprises, nous avons pu recapturer des truites radio-marquées et aucune nécrose ou infection n'a été observée au niveau de la zone d'incision, où la cicatrice était d'ailleurs souvent invisible. Ces observations suggèrent qu'au cours de cette étude, le comportement des truites suivies n'a pas été fortement perturbé par le marquage.

Ce travail montre clairement que, dans une petite rivière salmonicole à lit caillouteux comme l'Aisne, les patrons de mobilité et les stratégies d'occupation de l'espace des truites sont très variables au cours du cycle annuel. Les plus grands déplacements entre jours successifs sont observés pendant les mois d'octobre à décembre (Figure 6) pendant lesquels les truites effectuent leur migration de reproduction. Le mois d'octobre et la première quinzaine du mois de novembre correspondent essentiellement aux migrations vers l'amont. L'allure logarithmique des courbes de migration (Figure 3) suggère que les truites nagent le plus vite possible les premiers jours lorsque la température est encore élevée et donc probablement avantageuse sur le plan énergétique pour effectuer de longs déplacements.

En novembre et en décembre, tous les comportements propres à la reproduction (e.g. montaison, frai, dévalaison) peuvent être observés. La période de frai est étalée dans le temps, certains individus effectuant leur dévalaison alors que d'autres n'ont pas encore terminé leur migration vers l'amont. Quelques truites terminent leur dévalaison pendant la première quinzaine de janvier. D'autres études, réalisées dans des rivières françaises, ont montré que la période de frai de la truite débute en novembre et finit fin janvier, exceptionnellement fin février (EUZENAT et FOURNEL, 1976 ; BAGLINIERE et al., 1979 ; NIHOUARN, 1983 ; BAGLINIERE et al., 1989). Les études par radio-pistage sur les populations Nord-Américaines (Michigan, Wisconsin) montrent que les truites commencent leur migration de reproduction dès le mois de septembre (CLAPP et al., 1990 ; MEYERS et al., 1992 ; YOUNG, 1994), mais sous des conditions météorologiques assez différentes. Tous ces auteurs signalent aussi que les plus grands déplacements s'effectuent en automne et que des dévalaisons rapides se produisent après le frai chez $S$. trutta (MEYERS et al., 1992) et chez Salvelinus confluentus (SWANBERG, 1997). Dans notre 
étude, bien que peu de truites $(n=3)$ aient été suivies sur l'entièreté du cycle de reproduction, principalement à cause d'une durée de vie trop courte des batteries des émetteurs, toutes ont réalisé un homing post-reproducteur très précis, vers les gîtes occupés au printemps et en été. Ainsi, même si le phénomène n'est pas une règle générale, nos résultats suggèrent qu'il doit être assez fréquent.

Le reste de l'année, en février-septembre, les déplacements sont moins longs qu'en période de reproduction et correspondent principalement à des changements de gîtes dans un domaine vital bien défini dont l'étendue semble proportionnelle à la taille des individus. En mars et juin, certaines truites marquées au confluent de l'Aisne avec l'Ourthe effectuent toutefois de longues migrations unidirectionnelles (plusieurs kilomètres) vers l'amont qui sont parfois stoppées ou retardées par de petits déversoirs devenus infranchissables en période d'étiage. Suivant l'abondance des précipitations et les variations du niveau d'eau, la truite passe l'obstacle, ou au contraire, stoppe toute tentative de franchissement et finit par dévaler à quelques centaines de mètres en aval. La fonction biologique de ces migrations reste à préciser. Au mois de mars, elles pourraient correspondre à un retour tardif vers un gîte printanier après le frai dans une autre rivière. Au mois de juin, il pourrait s'agir d'un comportement de thermorégulation comportementale, les truites quittant l'Ourthe pour l'Aisne, dont le régime thermique se rapproche davantage de leur optimum $\left(T^{\circ} \leq 17^{\circ} \mathrm{C}\right.$; ELLIOTT, 1994) à cette période de l'année. MEYERS et al. (1992) montrent aussi qu'au printemps certaines truites effectuent des migrations vers l'amont qu'ils associent plus à des augmentations de température que de débit mais dont l'intérêt biologique est inconnu. Une étude réalisée dans l'Aisne (GOFFAUX, 1997) a montré que les truites qui vivent dans des environnements thermiques hétérogènes (zones de confluence) peuvent exprimer des comportements de thermorégulation comportementale qui se traduisent par l'utilisation successive de refuges thermiques s'approchant le plus du préférendum, en fonction des températures du cours principal et de l'affluent. II est intéressant de noter que les contrôles du piège de l'échelle à poissons de Bomal située sur l'Aisne à $500 \mathrm{~m}$ de la confluence avec l'Ourthe (Figure 1C), indiquent toujours un important pic de captures de truites au mois de juin (PHILIPPART et OVIDIO, études 1996-1998 en cours). Le rôle précis de ces migrations étant encore à déterminer, l'impact de faibles débits sur la biologie des populations de truite à ces périodes ne peut être précisé à l'heure actuelle.

Notre étude par biotélémétrie met en évidence que les truites montrent, en dehors de la période de reproduction, une mobilité plus élevée que ce qu'indiquent de nombreuses études par marquage-recapture (SCHUCK, 1945 ; TIMMERMANS, 1972 , 1976 ; SOLOMON et TEMPLETON, 1976 ; HARCUP et al., 1984 ; HESTHAGEN, 1988). Signalons que la précision de nos observations, et l'interprétation de leur causalité, n'auraient pu être possibles sans un positionnement journalier des truites, méthodologie trop rarement utilisée en biotélémétrie des poissons, du moins sur de longues périodes (BARAS, 1998).

De l'hiver à l'été, l'influence des facteurs environnementaux sur la mobilité des truites est très variable et individuelle. Ainsi, la variabilité des influences environnementales dépend probablement de la nature des gîtes et de l'aire d'activité où vit la truite, et, certainement aussi, de la variation de la capacité d'accueil des gîtes en fonction des modifications de plusieurs paramètres environnementaux tels que : vitesse de courant, profondeur, température, abondance de la nourriture, nature du milieu environnant (végétation, sous-berge).

Par contre, nous avons confirmé qu'en automne, les variables température et niveau d'eau exercent une action combinée précise sur le déclenchement des migrations de reproduction (voir OVIDIO et al., 1998 pour plus de détails). Le rôle favorable des forts débits, des crues et décrues sur les migrations de reproduction a déjà été mis en 
évidence dans des études antérieures (MUNRO et BALMAN, 1956 ; STUART, 1957 ; HUET, 1961 ; LIBOSVARSKY, 1976 ; HUET et TIMMERMANS, 1979). Dans le cas de débits moyens, d'autres auteurs ont montré que les basses températures dans le ruisseau $\left(<6^{\circ} \mathrm{C}\right)$ semblent jouer un rôle inhibiteur (EUZENAT et FOURNEL, 1976 ; BAGLINIERE et al.,1987, MAISSE et BAGLINIĖRE, 1991).

La possibilité de prévoir les moments précis où les truites vont débuter leur migration de reproduction est particulièrement intéressante pour la gestion des populations:- Elle pourrait permettre, par exemple, de modifier les débits ou d'ouvrir momentanémént certains barrages, normalement infranchissables, lors des vagues de migration. Mais de nombreuses mises au point techniques sont encore nécessaires pour que les ouvertures de barrages ou les appels d'eau par éclusée puissent réellement être opérationnels. Des éclusées bien programmées pourraient alors avoir un impact favorable sur les populations de truites alors qu'habituellement, les poissons sont fortement perturbés par ces pratiques (CRISP, 1993 ; MOOG, 1993 ; LIEBIG, 1994 ; LAUTERS, 1995 ; VALENTIN, 1995 ; LIEBIG et al., 1996).

Au moment de la reproduction, les distances parcourues sont importantes et de très petits affluents sont empruntés. Il est donc biologiquement indispensable de permettre une libre circulation des truites dans l'ensemble du bassin hydrographique.

\section{REMERCIEMENTS}

Pour la réalisation d'un doctorat sur l'écologie comportementale de la truite commune, j'ai pu bénéficier d'une bourse du FRIA (Fond pour la Formation à la Recherche dans I'Industrie et l'Agriculture). Je remercie les Drs J.C. PHILIPPART, promoteur de thèse, et E. BARAS (co-promoteur) ainsi que deux lecteurs anonymes, pour leurs suggestions et remarques très constructives à la lecture d'une version préliminaire du manuscrit. Pour les nombreuses heures passées sur le terrain et leur efficacité, j'éxprime tout spécialement ma reconnaissance à D. GOFFAUX, F. GIROUX, G. RIMBÄUD et C. BIRTLES. Je tiens également à remercier D. PARKINSON, B. SEROUGE, D. JEANDRAIN, G. QUINCHON et $\mathrm{C}$. BRUCY pour leur aide précieuse ainsi que les responsables et membres de la Société de Pêche de l'Aisne, J. BLAUDE et Mr DENIS pour leur aimable collaboration. Cette recherche s'inscrit dans le projet "Meuse Saumon 2000 " financé par le Ministère de la Région Wallonne (Ressources Naturelles et Environnement) et coordonné à l'Université de Liège par J.C. PHILIPPART (chercheur qualifié au FNRS, Fonds National de la Recherche Scientifique).

\section{BIBLIOGRAPHIE}

ALLENDORF F., 1996. Genetic and demographic effects of fragmentation and isolation of fish populations. Communication à : International Conference on Fish Migration and Fish Bypass-Channels, 24-26 septembre 1996, Vienne, Autriche, 9 p.

BAGLINIERE J.L., CHAMPIGNEULLE A., NIHOUARN A., 1979. La fraie du saumon atlantique (Salmo salar) et de la truite commune (Salmo trutta L.) sur le bassin du Scorff. Cybium, $3^{\circ}$ série, 7, 75-96.

BAGLINIĖRE J.L., MAISSE G., LEBAIL P.Y., PREVOST E., 1987. Dynamique de la population de truite commune (Salmo trutta L.) d'un ruisseau breton (France). II. Les géniteurs migrants. Acta CEcologica CEcol. Applic., 3, 201-215. 
BAGLINIĖRE J.L., MAISSE G., LE BAIL P., NIHOUARN A., 1989. Population dynamics of brown trout, Salmo trutta L., in a tributary in Brittany (France) : spawning and juveniles. J. Fish Biol., 34, 97-110.

BAGLINIÈRE J.L., MAISSE G., (eds), 1991. La truite : biologie et écologie. INRA Editions, Paris, $303 \mathrm{p}$.

BARAS E., 1992. Etude des stratégies d'occupation du temps et de l'espace chez le barbeau fluviatile, Barbus barbus (L). Cah. Ethol. Appl., 12, 125-442.

BARAS E., 1998. Selection of optimal positioning intervals in fish tracking : an experimental study on Barbus barbus. Hydrobiologia, 1998.

BARAS E., BIRTLES C., WESTERLOPPE L., THOREAU X., OVIDIO M., JEANDRAIN D., PHILIPPART J.C., in press. A critical review of surgery techniques for implanting telemetry devices into the body cavity of fish. In Le Maho Y. (ed.), Proceedings of the 5th European Conference on Wildlife Telemetry, Strasbourg (France), 26-30 August 1996, $10 \mathrm{p}$.

BIRTLES C., BARAS E., PONCIN P., GOESSENS G., PHILIPPART J.C., 1995. A behavioural and histological assessment of post-tagging stress in brown trout Salmo trutta L. equipped with surgicaly implanted biotelemetry transmitters. In Abstract book of the Second Benelux Congress of Zoology, Leiden (Holland), November 18-19, 1995.

CLAPP D.F., CLARK R.D., DIANA J.S., 1990. Range, activity and habitat of large, free-ranging brown trout in a Michigan Stream. Trans Am. Fish. Soc., 119, 10221034.

CRISP D.T., 1993. Populations densities of juvenile trout (Salmo trutta) in five uplands stream and their effects upon growth, survival and dispersal. Journal of Applied Ecology, 30, 759-771.

ELLIOTT J.M., 1994. Quantitative ecology and the brown trout. Oxford University Press, Oxtord, $286 \mathrm{p}$.

EUZENAT G., FOURNEL F., 1976. Recherche sur la truite commune (Salmo trutta L.) dans une rivière de Bretagne, le Scorff. 1. Caractéristiques démographiques des populations de truite commune de la rivière Scorff et des affluents. 2. Premiers éléments d'une étude de dynamique des populations de truite commune. Thèse de Doctorat 3ème cycle, Biol. Anim. Fac. Sci. Univ., Rennes, 213 p.

FAUSH K.D., YOUNG M.K., 1995. Evolutionarily Significant Units and Movement of Resident Stream Fishes : A Cautionary Tale. American Fisheries Society Symposium, 17, 360-370.

GOFFAUX D., 1997 Incidence du régime thermique sur l'utilisation du temps et de l'espace par la truite commune (Salmo trutta L.) dans une rivière salmonicole, l'Aisne. Mémoire de Licence en Sciences Biologiques, LDPA-Université de Liège, $50 \mathrm{p}$.

GOWAN C., YOUNG M.K., FAUSCH K.D., RILEY S.C., 1994. Restricted movements in resident stream salmonids : a paradigm lost. Can. J. Fish. Aquat. Sci., 51, 2626-2637.

GUYOMARD R., KRIEG F., 1986. Mise en évidence d'un flux génétique entre populations naturelles de truites fario et souche de repeuplement dans deux rivières de Corse. Bull. Fr. Pêche Piscic., 303, 134-140.

HARCUP M.F., WILLIAMS R., ELLIS D.M., 1984. Movements of brown trout, Salmo trutta L., in the River Gwyddon, South Wales. J. Fish Biol., 24, 415-426.

HAURY J., OMBREDANE D., BAGLINIERE J.L., 1991. L'habitat de la truite commune (Salmo trutta) en cours d'eau. In Baglinière J.L. et Maisse G. (eds), La truite : biologie et écologie, 48-96, INRA Publ., Paris. 
HESTHAGEN T., 1988. Movements of brown trout, Salmo trutta, and juvenile Atlantic salmon, Salmo salar, in a coastal stream in northern Norway. J. Fish Biol., 32, 639-653.

HUET M., 1949. Aperçu de la relation entre la pente et les populations piscicoles des eaux courantes. Schweiz. Z. Hydrol., 11, 332-351.

HUET M., 1961. Reproduction et migrations de la truite commune (Salmo trutta fario L.) dans un ruisselet salmonicole de l'Ardenne belge. Verh. int. Ver. Limnol., 14, 757-762.

HUET M., TIMMERMANS J.A., 1979. Fonctionnement et rôle d'un ruisseau frayère à truites. Station de Recherches...Eaux et Forêts Groenendaal-Hoeilaart, Travaux-série $D, N^{\circ} 48,31 p$.

JAMES G.D., KELSO J.R.M., 1985. Movements and habitat preference of adult rainbow trout (Oncorhynchus mykiss) in a New Zealand montane lake. Nëw Zealand J. Mar. Freshw. Res.; 29, 493-503.

LARINIER M., PORCHER F., TRAVADE F., GOSSET C., .1995. Passes à poissons. Expertise et conception des ouvrages de franchissement: Collection "Mise au point ". Conseil Supérieur de la Pêche, Paris, France, 336 p.

LAUTERS F., 1995. Impacts sur l'écosystème aquatique de la gestion par éclusées des ouvrages hydroélectriques. Thèse de Doctorat, Université Paul Sabatier de Toulouse, $188 \mathrm{p}$.

LIBOSVARSKY J., 1976. On the ecology of spawning migration of brown trout. Zool. Listy., $25,175-182$.

LIEBIG H., 1994. Etude prélimainaire de la population de truite commune Salmo trutta fario sur l'Oriege. Influence des éclusées. Rapport de DEA, Université Paul Sabatier de Toulouse, $42 \mathrm{p}$.

LIEBIG H., LIM P., BELAUD A., LEK S., 1996. Study of the juvenile community in the brown trout (Salmo trutta fario L., 1758) in hydropeaking situations. In Leclerc M. et al. (eds), Ecohydraulics 2000, Proceedings of the Second IAHR Symposium on Habitat Hydraulics, INRS-Eau, Vol. A, 673-684.

MAISSE G., BAGLINIERE J.L., 1991. Biologie de la truite commune. In Baglinière J.L. et Maisse G. (eds), La truite : biologie et écologie, 25-45, INRA Publ., Paris.

MALAVOI J.R., 1989. Typologie des facies d'écoulement ou unités morpho-dynamiques des cours d'eau à haute énergie. Bull. Fr. Pêche Piscic., 315, 189-210.

MEYERS L.S., THUEMLER T.F., KORNELY G.W, 1992. Seasonal movements of brown trout in Northeast Wisconsin. N. am. J. Fish. Manage., 12, 433-441.

MOOG O., 1993. Quantification of daily peak hydropower effects on aquatic fauna and management to minimize environmental impacts. Regul. Riv. : Res. \& Manage., 8, 5-14.

MUNRO W.R., BALMAN K.H., 1956. Observations on the spawning runs of brown trout in the South Queich, Loch Leven. Freshwat. Salm. Fish. Res.; 13, 17 p.

NIHOUARN A., 1983. Etude de la truite commune (Salmo trutta L.) dans le bassin du Scorff (Mơrbihan) : démographie, reproduction, migration. .Thèse de Doctorat 3ème cycle, Biol. Anim. Fac. Univ., Rennes, 73 p.

NORTHCOTE T.G., 1967. The relation of movements and migration to production in freshwater:fish. In Gerking S.B. (ed.), The Biological Basis of Freshwater Fish Production; 315-344, Blackwell, Oxford. 
OVIDIO M., BIRTLES C., BARAS E., PHILIPPART J.C., 1996. A preliminary telemetry investigation on the obstacles to anadromous Salmonids migration in spawning streams of the Belgian Ardennes (river Meuse basin). In M. Leclerc et al. (eds), Ecohydraulics 2000, Proceedings of the Second IAHR Symposium on Habitat Hydraulics, Vol. A, 83-88, INRS-Eau.

OVIDIO M., BIRTLES C., BARAS E., PHILIPPART J.C., GIROUX F., 1999. Daily and seasonal movements of radio-tagged brown trout (Salmo trutta) in the Belgian Ardennes: mobility versus residency. In Le Maho Y. (ed.), Proceedings of the Fifth European Conferences on Wildlife Telemetry in Europe, Strasbourg (France), 25-30 August 1996, 6 p., in press.

OVIDIO M., BARAS E., GOFFAUX D., BIRTLES C., PHILIPPART J.C., 1998. Environmental unpredictability rules the spawning migrations of trout (Salmo trutta) in the Belgian Ardennes. Hydrobiologia, 371/372, 263-274.

PHILIPPART J.C., 1975. Dynamique des populations de poissons non exploités. In Lamotte $M$. et Bourlière F. (eds), Problèmes d'écologie. Dynamique des populations de vertébrés, 291-394, Masson, Paris, $443 \mathrm{p}$.

PHILIPPART J.C., 1987. Histoire de l'extinction et problématique de la restauration des salmonidés migrateurs dans la Meuse. In Thibault M. et Billard R. (eds), La restauration des rivières à saumons, Collection Hydrobiologie et Aquaculture, Publication, 125-137, INRA Paris.

PHILIPPART J.C., 1998. Ecologie de la truite commune (Salmo trutta L.) dans les rivières de Wallonie. Ch. 5, Démographie et Dynamique des populations. Rapport de recherche du Laboratoire de Démographie des Poissons et d'Aquaculture, Université de Liège, 54 p. (Version de janvier 1998).

PHILIPPART J.C., MICHA J.C., BARAS E., PRIGNON C., GILLET A., JORIS S., 1994. The Belgian project «Meuse Salmon 2000 ». First results, problems and future prospects. Water Sci. Technol., 29, 315-317.

PHILIPPART J.C., RIMBAUD G., BIRTLES C., OVIDIO M., GIROUX F., GOFFAUX D., JENDRAIN D., BARAS E, 1997. Convention d'étude pour le suivi scientifique de la réintroduction du Saumon atlantique dans le bassin de la Meuse. Projet "Meuse Saumon 2000 ". Rapport pour le Ministère de la Région Wallonne (DGRNE) pour la période février 1996-janvier 1997, 112 p. + annexes.

SCHUCK H.A., 1945. Survival, population, density, growth, and movement of wild brown trout in crystal Creek. Trans. Am. Fish. Soc., 73, 209-230.

SOLOMON D.J., TEMPLETON R.G., 1976. Movements of brown trout in a chalk stream. J. Fish Biol., 9, 411-423.

STUART T.A., 1957. The migration and homing behaviour of brown trout (Salmo trutta L.) Freshwat. Salm. Fish. Res., 18, 27 p.

SWANBERG T.R., 1997. Movements of and habitat use by fluvial bull trout in the Blackfoot River, Montana. Transactions of the American Fisheries Society, 126, 735-746.

TIMMERMANS J.A., 1972. La territorialité de la truite fario. Trav. Stat. Rech. Eaux et Forêts Groenendaal, Série D, $n^{\circ} 42,15 p$.

TIMMERMANS J.A., 1976. Attachement et retour de la truite fario (Salmo trutta fario L.) à son habitat. Trav. Stat. Rech. Eaux et Forêts Groenendaal, Série $D, n^{\circ} 45,19 p$.

VALENTIN S., 1995. Variabilité artificielle des conditions d'habitat et conséquence sur les peuplements aquatiques : effets écologiques des éclusées hydroélectriques en rivière. Thèse de Doctorat, Université Claude Bernard de Lyon 1, $275 \mathrm{p}$.

YOUNG M.K., 1994. Mobility of brown trout in south-central Wyoming streams. Can. J. Zool., 72, 2078-2083. 